\title{
CAMBIO DE PATRONES DE CIRCULACIÓN DE VERANO Y OTOÑO EN LA PENÍNSULA IBÉRICA DURANTE LAS DOS RECIENTES FASES DE LA ATLANTIC MULTIDECADAL OSCILLATION
}

\author{
Alejandro LOMAS GONZÁLEZ1 ${ }^{1}$, Ricardo TORRIJO MURCIANO ${ }^{1}$ \\ ${ }^{1}$ Agencia Estatal de Meteorología. \\ alomasg@aemet.es, rtorrijom@aemet.es
}

\section{RESUMEN}

Entre los años 1960 a 2015 la temperatura media de verano experimentó en la Península ibérica una subida superior a $1^{\circ} \mathrm{C}$, superior a la que cabe esperar por el calentamiento global, por lo que cabe indagar en el aporte propio de la variabilidad climática que puede inducir cambios de patrón de circulación. De la misma forma, la precipitación otoñal en los observatorios del oeste peninsular ha registrado un aumento. También se puede asociar a un cambio de patrón que pudiera guardar relación con el de verano.

La hipótesis de trabajo principal es que el debilitamiento del anticiclón de Azores conduce no solo a un aumento de las temperaturas de verano, sino también a un adelanto del mismo que se manifiesta en un ascenso de las temperaturas de junio. Cambios similares se aprecian en la siguiente estación, el otoño. Se investiga esa relación en tres fases: análisis de la temperatura de verano y de la precipitación de otoño de los últimos 55 años de varios observatorios españoles, posterior estudio sobre los cambios de circulación atmosférica asociada con los cambios significativos y por último la posible conexión con la AMO y la actividad solar.

Palabras clave: AMO, actividad solar, temperatura verano, precipitación otoño, cambios circulación general atmosférica.

\begin{abstract}
Between 1960 and 2015, the summer average temperature increased more than the amount expected due to global warming $\left(1^{\circ} \mathrm{C}\right)$. The role of climate variability, with its associated changes in global circulation, should be taken into account to explain this fact. The observed rise of the autumn precipitation in western Iberian Peninsula can also be associated with similar changes to those observed in summer.

The main hypothesis is that the weakening of Azores high can lead, not only to an increase in summer temperature, but also to a sooner summer entering, that can be observed in the rise of June temperatures. Similar changes in global circulation are also observed in autumn. The research will be made in three steps: analysis of summer temperature and autumn precipitation during the last 55 years in different Spanish observatories, analysis of changes in circulation patterns that can be associated with significant changes and, finally, its possible connexion with AMO and solar activity.
\end{abstract}


Keywords: AMO, solar activity, summer temperature, autumn precipitation, changes in global atmospheric circulation .

\section{INTRODUCCIÓN}

En el contexto de preocupación por el aumento de la temperatura planetaria registrado durante el último siglo, debido posiblemente a un incremento de la concentración en la atmosfera de gases invernadero y/o a la mera variabilidad climática, cabe preguntarse que parte en ese aumento tiene cada uno de esos dos factores. Según la propia definición del Panel Intergubernamental para el cambio climático: "Cada una de las últimas tres décadas ha sido sucesivamente más cálidas en la superficie de la Tierra que cualquiera de las precedentes desde 1850. En el Hemisferio Norte, 1983-2012 fue probablemente el período de 30 años más cálido de los últimos 1400 años" (IPCC, 2013).

La intención de hacer éste trabajo se apoya en:

- En la Península Ibérica “Al igual que sucede con la temperatura global, dentro de la tendencia general a la alza destaca un breve periodo (1950-1972) en el cual las temperaturas anuales no aumentaron o incluso disminuyeron ligeramente, aunque no de forma significativa. De las dos fases más cálidas observadas en el siglo XX (1901-1949 y 1973-2005), es en la más reciente cuando se han producido las tasas de calentamiento más elevadas". En este período "se han llegando a alcanzar tasas de $0.5^{\circ} \mathrm{C} /$ década (...) que casi triplican el ritmo de aumento de la temperatura media global. Estacionalmente, a lo largo del siglo XX, todas las estaciones han contribuido al incremento anual, con tasas de cambio similares. En la reciente fase de calentamiento, sin embargo, las estaciones que más han contribuido han sido la primavera y el verano" (CLIVAR, 2010).

- Entre 1971-2000 y 1981-2010 la temperatura se incrementó 0,46 ${ }^{\circ} \mathrm{C}$ en España, más del doble que la observada entre los dos períodos de referencia anteriores, $1961-1990$ y $1971-2000$, que fue de $0,22^{\circ} \mathrm{C}$. Esto es más visible aún en los veranos tanto en lo que respecta al aumento de la temperatura como en su adelanto, con junios más cálidos (Lomas et al., 2014).

- Por otro lado la evolución hacia temperaturas más altas no es continua, sino que se perciben dos anomalías, una fría en los setenta y otro cálida al principio del siglo XXI.

- Además se observan en la evolución de las temperaturas de verano dos períodos bien diferenciados (1960-1990/1991-2015) con poca tendencia dentro de ellos, un grado más cálido el segundo. Sugieren dos estados o patrones climáticos diferentes y se aproximan a la secuencia negativa-positiva de la Atlantic Multidecadal Oscillation (AMO) cuya influencia en el clima europeo es destacable (véase por ejemplo Sutton et al., 2012.). Teniendo en cuenta los dos puntos anteriores se buscan explicaciones a través de cambios de la circulación general atmosférica que justifiquen al menos parte de ese aumento extra y posibles relaciones con la AMO.

- Los patrones más sencillos de la circulación en verano facilitan el análisis.

- Se investigará la posible continuidad o discontinuidad de dichos cambios en la circulación de otoño que pudieran dar lugar a cambios de patrón de la precipitación. Estudios realizados parecen indicar cambios en dicho patrón de precipitación en la Península (De Castro et al., 2005), así como una tendencia al cambio de su distribución estacional. 


\section{MÉTODO}

El trabajo que se ha desarrollado se ha elaborado en tres fases:

1.- Un análisis de los datos de temperatura de verano y precipitación de otoño de Observatorios de AEMET de Burgos, La Coruña, Navacerrada, Retiro, Sevilla-Morón, Valencia, Valladolid y Zaragoza desde 1960 hasta 2015. Se ha elegido ese período por disponer de datos suficientemente buenos y comparables al reanálisis de NCEP, cuyos datos llegan hasta los años 50 (Kalnay et al., 1996). Para hacer el análisis se centrará sobre 2 períodos bien diferenciados: 1964-89 y 1990-2015 en los que se observan diferencias importantes y además se corresponden con fases opuestas de la AMO. El puro análisis de los datos se hace en paralelo con los dos patrones de comportamiento dinámico de la atmósfera. Sin ese estudio paralelo carecería de sentido dicho análisis.

2.- También para esos dos períodos citados se parte del reanálisis del NCEP para estudiar las variaciones de la circulación general atmosférica asociadas y que podrían explicar, al menos parte, los cambios. Se utilizan los niveles de superficie, 850, 500 y $300 \mathrm{hPa}$.

3.- Partiendo de las conclusiones de los puntos 1 y 2 se intenta relacionar y explicar esos cambios de temperatura con el contexto del cambio climático, variabilidad interna del sistema climático (AMO) y variaciones en la actividad solar.. También se investiga la evolución de las precipitaciones de otoño en los anteriores observatorios. En la zona oeste peninsular se perciben dos períodos "anómalos": años de predominio de otoños secos (en torno a los setenta y principio de los ochenta del siglo pasado) y otra de predominio de otoños húmedos (al principio del siglo XXI). Parecen indicar dos períodos con estados o patrones climáticos diferentes y se ha utilizado también el reanálisis del NECP, para estudiar las variaciones de la circulación y poder inferir las causas y también las concomitancias con la circulación de verano

\section{RESULTADOS}

\subsection{Patrones sinópticos característicos de los verano de la Península}

La presencia del Anticiclón centrado en torno a las islas Azores, que se extiende más o menos en forma de cuña sobre el norte de la Península, y la de la baja debilitada en las cercanías de Islandia es la situación más característica del verano. La cuña anticiclónica suele avanzar y retroceder sobre la Península, desalojando o permitiendo el desarrollo de la baja y dorsal térmica del norte de África y del sur de España. El juego de todos estos sistemas de presión hace que el verano de la Península sea más o menos caluroso. Cuanto más influencia hay de la componente norte, más fresco será el verano y si esa influencia no es importante se forma una gran masa cálida sobre la Península. Estos patrones son generalizados para todos los "julios" y "agostos", por lo que en éste trabajo, por esta y alguna razón más, se considerarán en momentos aparte del mes de junio, mes en el que es habitual el mantenimiento de una circulación atmosférica primaveral, aunque debilitada por lo general.

Por otro lado las síntesis del IPCC ya determinan que se detecta un debilitamiento del anticiclón de Azores en los últimos 30 años. Además cabe esperar, con un aumento de temperatura natural o antropogénico, el ascenso en latitud de los sistemas de presión subtropicales. 


\subsection{Temperaturas de verano en observatorios en los dos períodos}

Se han elegido estaciones representativas de distintas zonas de la península. Dado que se pretende buscar cambios en la circulación atmosférica no es necesario extrapolar geográficamente para hacer un estudio de la zona completa, sino observar los patrones de temperatura que se van a inferir de distintas situaciones sinópticas.

El patrón que se observa para el observatorio de Burgos (Fig 1) es similar al de los otros observatorios pero con matices mucho más puros. Se pueden resumir en las siguientes características:

- Por otro lado la evolución hacia temperaturas más altas no es continua, sino que se perciben dos anomalías, una fría en los setenta

- Tendencia claramente ascendente en los 54 años con dos períodos, 1964-1989 y 1990-2015 sin tendencias o con poca tendencia para cada uno.

- Esos dos períodos parecen obedecer a patrones de tiempo diferente, uno más fresco y otro claramente más cálido

- Dos anomalías, una fría en torno a los años setenta y otra cálida al principio del siglo XXI.

- Esa anomalía cálida es mucho más clara en los junios de la primera década del siglo XXI, donde los "junios" son casi tan cálidos como los "julios" y "agostos", aunque parece desvanecerse éstos últimos años esa circunstancia.

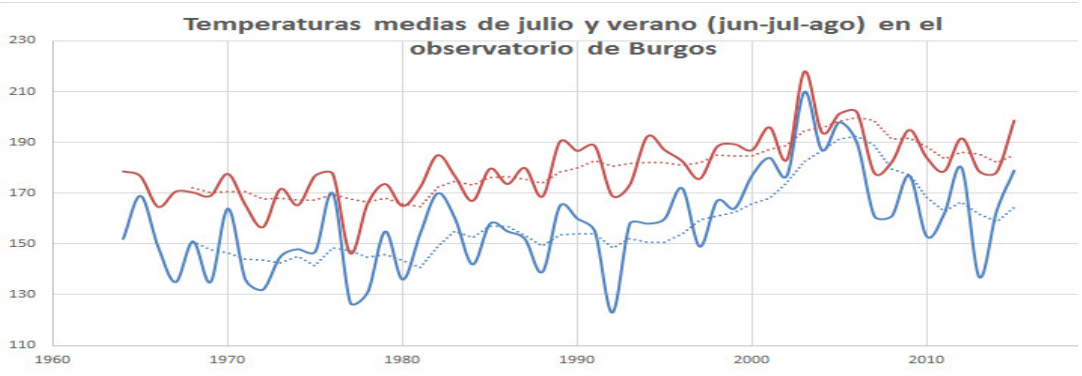

Fig.1: Temperaturas de junio y veráno para el observatorio de Burgos. Fuente: Banco Nacional de Datos Climatológicos de AEMET

\begin{tabular}{|l|c|c|c|}
\hline \multicolumn{4}{|c|}{ Temperaturas medias de verano en los dos períodos en décimas de ${ }^{\mathbf{0}} \mathbf{C}$} \\
\hline Estación & $1964 / 1989$ & $1990 / 2015$ & Diferencia \\
\hline Burgos & 172 & 188 & 16 \\
\hline Retiro & 229 & 247 & 18 \\
\hline Navacerrada & 144 & 160 & 16 \\
\hline Valencia & 234 & 252 & 18 \\
\hline Zaragoza & 230 & 247 & 17 \\
\hline Sevilla/Morón & 246 & 261 & 15 \\
\hline
\end{tabular}

Tabla 1: Temperaturas medias (décimas de ${ }^{\circ} \mathrm{C}$ ) de verano en dos períodos para varios observatorios. Fuente: Banco Nacional de Datos de AEMET. 
En el cuadro anterior (Tabla 1), las diferencias alcanzan o superan $1.5^{\circ} \mathrm{C}$ entre los períodos 1964/1989 y 1990/2015, tanto en conjunto como consideradas mes a mes, destacando junio y agosto como los que contribuyen más a la subida. En la comparación entre la década más cálida, 2000-2009, y la más fría, 1970-1979, los valores se acercan o alcanzan $\operatorname{los} 4^{\circ} \mathrm{C}$ de diferencia.

Las diferencias básicas se pueden justificar por una de las siguientes causas o por ambas a la vez:

- El aumento de la concentración de los gases llamados de invernadero, aunque sería insuficiente explicación dado que el ritmo de subida es bastante mayor.

- Un cambio en las configuraciones sinópticas que afecten a la península de forma que favorezcan en la misma una subida de temperaturas.

En caso de que ambas circunstancias actuaran a la vez sería imposible determinar cuánto peso dar a cada una, aunque se podría hacer una valoración subjetiva.

\subsection{Razones sinópticas que favorecen junios más fríos o más cálidos}

Una vez evaluadas y cuantificadas las variaciones de temperatura de los meses de verano, se procederá a la siguiente etapa del trabajo en el que, a través del reanálisis del NCEP, se buscarán configuraciones sinópticas que expliquen ya definitivamente esos cambios de temperatura entre los períodos 1964-1989 y 1990-2015. Para ello se utilizarán básicamente los niveles de superficie, 850, 500 y $300 \mathrm{hPa}$.

La configuración favorecedora de temperaturas más bajas en verano implica una circulación en capas bajas con más frecuencia de componente norte. Esto se produce con un reforzamiento del anticiclón de Azores que además sube en latitud, mientras que las presiones sobre Europa en los períodos de veranos más fríos son algo más bajas (Fig. 2)
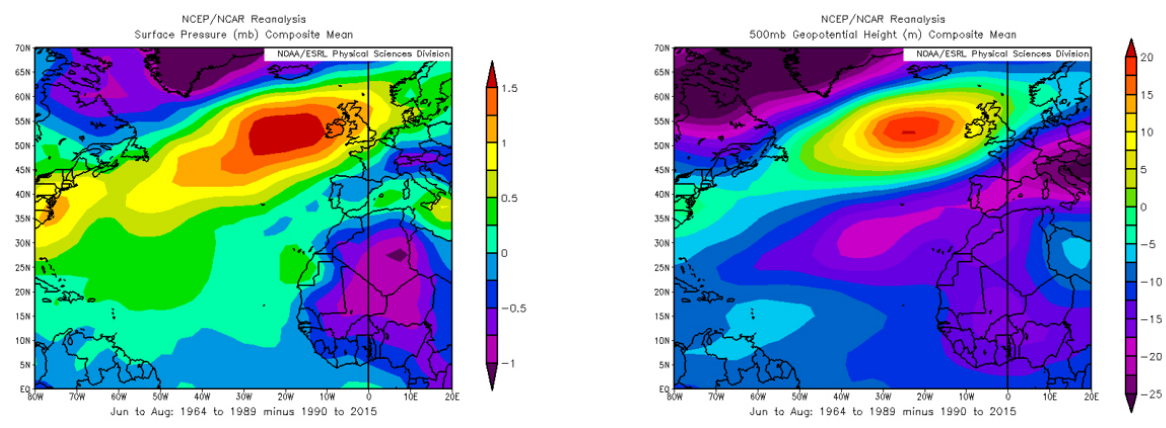

Fig 2: Diferencias de presión en superficie (izda) y de geopotencial en $500 \mathrm{hPa}$ (dcha) entre los dos periodos considerados 1964/1989 y 1990/2015. Fuente: Reanálisis tomado de NOAA/ OAR/ESRL PSD, Boulder, Colorado, USA, de su página web en http://www.esrl.noaa.gov/psd/

Estos cambios en la circulación se reflejan en la circulación y en los cambios de temperatura. El resultado es una clara subida de temperaturas en la Península con centro en el sureste asociado a unas condiciones de forzamiento que generan una masa de aire cálido sobre la Península que no es aliviada con entradas de componente norte. 
La última aproximación es observar lo que ocurre con la posición de los máximos en $300 \mathrm{hPa}$ y los cambios en los flujos viendo las diferencias entre los dos períodos (Fig. 3). En una visión regional se aprecian tres circunstancias:

- Disminución de la circulación del oeste en el entorno atlántico de Islandia durante el segundo período.

- Correspondiente aumento en la latitud de las Islas británicas que afecta a la Península con circulación más zonal en el segundo período.

- Disminución de la intensidad del chorro subtropical
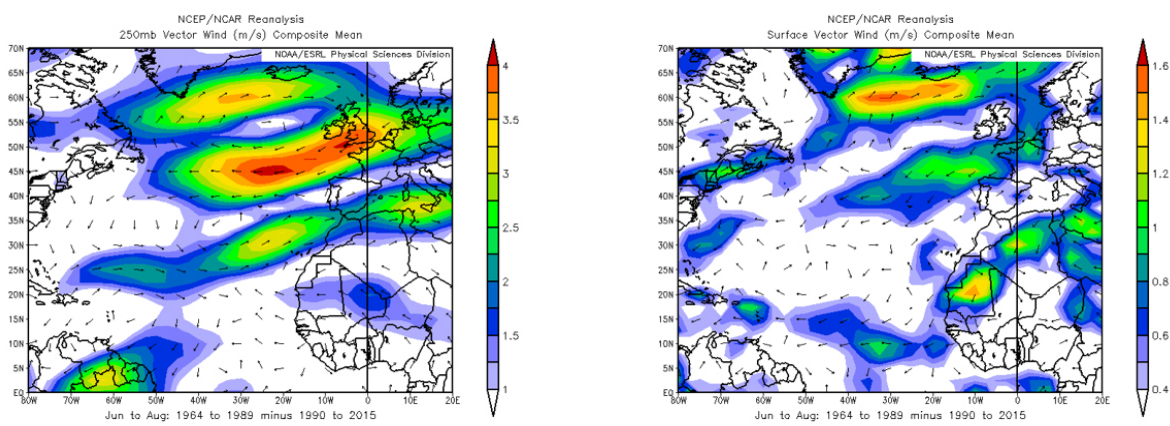

Fig 3: Diferencias de vector viento en 300 hpa (izda) y en superficie (dcha) entre los dos períodos considerados 1964/1989 y 1990/2015. Fuente: Reanálisis tomado de NOAA/OAR/

ESRL PSD, Boulder, Colorado, USA, de su página web en http://www.esrl.noaa.gov/psd/

En resumen se puede ver una disminución en latitud de las corrientes del oeste, es decir mayor zonalidad en latitudes cercanas a nuestra Península, aunque muy debilitada por ser verano, a la vez que disminuye a la altura de Islandia. También en el entorno de nuestra Península se observa un cambio a situaciones con menos componente norte favoreciendo la instalación y persistencia de una masa de aire cálido.

\subsection{Cambio de circulación en otoño que continúan la de verano}

Se expondrán sólo las conclusiones del trabajo presentado en el último congreso de la Asociación Meteorológica Española en Teruel el pasado mes de marzo (Lomas et al., 2016):

- Hay un aumento de la precipitación en otoño en el oeste de la Península durante los últimos 25 años con respecto a los anteriores 25 años.

- Ese aumento es más significativo cuanto más al oeste sugiriendo un cambio de circulación favorecedora para el flujo de componente oeste.

- El aumento de la precipitación correlaciona con la fase de AMO positiva.

- Los cambios de circulación encajan con el cambio detectado de intensidad de la corriente en chorro. A la par que se produce un debilitamiento de la circulación del chorro en su latitud habitual, se produce el aumento correspondiente en las latitudes peninsulares.

- Todo encaja con cambios de la circulación que favorecen en los últimos años el descenso en latitud de la circulación tanto en verano como en otoño. 


\subsection{Relaciones entre AMO y las temperaturas de verano en España}

El observado patrón de variación multidecadal de la temperatura superficial del Atlántico Norte, es conocido como AMO (del inglés Atlantic Multidecadal Oscillation). Los registros instrumentales muestran como "en relación a la temperatura media del resto de océanos del mundo, la temperatura del Atlántico norte ha fluctuado entre fases anormalmente cálidas y frías, cada una de ellas de varias décadas de duración. Los registros paleoclimáticos muestran además que variaciones similares pueden llevarse muy atrás en el tiempo" (Sutton et al., 2012).

En estos momentos y desde principio de los años noventa, cuando comenzó tras 30 años de fase fría, se está en la fase cálida de la AMO. En la tabla 2 se ha calculado el índice de correlación de la temperatura de verano con la AMO y en la figura 4 se observa la relación de ese índice con la temperatura de verano de Burgos.

\begin{tabular}{|c|c|c|c|c|c|}
\hline \multicolumn{5}{|c|}{ Correlación temperatura de verano de varios observatorios peninsulares-AMO } \\
\hline Burgos & La Coruña & Sevilla & Valladolid & Retiro & Navacerrada \\
\hline 0,62 & 0,67 & 0,67 & 0,52 & 0,60 & 0,59 \\
\hline
\end{tabular}

Tabla 2: Índices de correlación entre la temperatura media de verano de varios observatorios peninsulares y la AMO. Fuente: Banco Nacional de Datos Climatológicos de AEMETy Reanálisis tomado de NOAA/OAR/ESRL PSD, Boulder, Colorado, USA, de su página web en http://www.esrl.noaa.gov/psd/

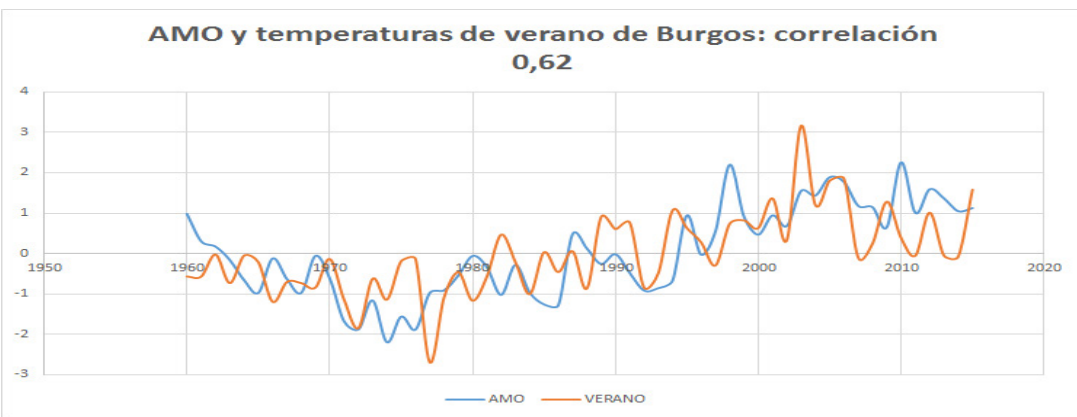

Fig 4: Representación de los valores medios normalizados anuales del indice AMO y las temperaturas de verano del observatorio de Burgos. Fuente: Banco Nacional de Datos Climatológicos de AEMET y Reanálisis tomado de NOAA/OAR/ESRL PSD, Boulder, Colorado, USA, de su página web en http://www.esrl.noaa.gov/psd/

\subsection{Relaciones entre el AMO y la intensidad de los vientos alisios en $10-20^{\circ} \mathrm{N}$}

También se ha encontrado, con datos desde 1960, una interesante correlación de la AMO con la intensidad de los vientos alisios, dando a entender una completa interacción entre todos los actores de la circulación del Atlántico norte (Fig. 5). El aumento de la intensidad de los alisios en las fases frías es coherente, por el principio de la conservación del momento, con una disminución de la circulación del oeste en latitudes más altas, tal y como se observa. 


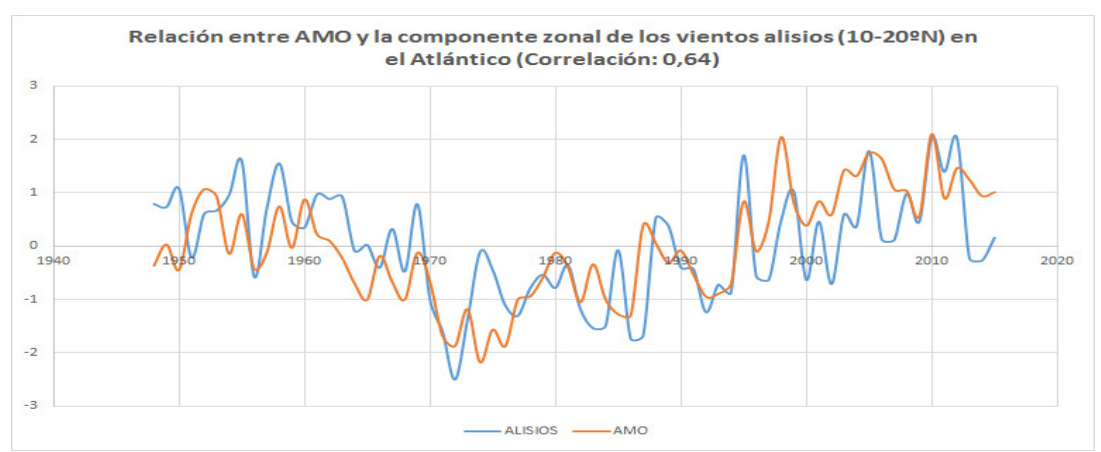

Fig 5: Representación de los valores medios normalizados anuales del índice AMO y la intensidad de la componente zonal de los vientos alisios en el Atlántico. Fuente Reanálisis tomado de NOAA/OAR/ESRL PSD, Boulder, Colorado, USA, de su página web en http://www. esrl.noaa.gov/psd/

\section{DISCUSIÓN}

Se han planteado cambios en los patrones de circulación relativos a cambios en la actividad solar. Por ejemplo Wooling, et al., en 2010, encontraron una clara correlación con situaciones atmosféricas de bloqueo anticiclónico en latitudes medias altas que provocan inviernos fríos en Europa y la oscilación NAO que genera inviernos templados. (Wooling et al, 2010)

Se explora la hipótesis solar para explicar los cambios de circulación y/o la variación de las temperaturas de la superficie del mar (AMO). Varios autores han relacionado recientemente la actividad solar con las variaciones de la circulación atmosférica. Sara Ineson (Ineson et al., 2011), propone que la baja actividad solar puede contribuir a los fríos inviernos en Europa occidental y América del Norte. El mecanismo meteorológico lo plantean los autores de la siguiente manera: en años de baja radiación UV, aire más frío se forma en la estratosfera tropical, a más de $50 \mathrm{~km}$ de altitud. Como los mecanismos atmosféricos son un juego de equilibrios, esto se compensa con un mayor flujo del Este sobre las latitudes medias, trayendo aire más frío a ciertas zonas como es el norte de Europa.

El experimento SORCE parece haber revelado que las diferencias en radiación UV durante el ciclo solar es mayor de lo esperado. Las medidas por satélite con el Spectral Irradiance Monitor (SIM) muestran que la variabilidad en el ultravioleta sobre la parte descendente del ciclo solar 23 puede ser considerablemente más grande que lo indicado por anteriores estimaciones (Haigh et al, 2010).

Es de particular importancia la radiación solar en el ultravioleta extremo (UVE), la cual alcanza su punto de mayor intensidad durante los años cercanos al máximo solar. Dentro de la relativamente estrecha banda de las longitudes de onda del UVE, la producción solar varía no por un minúsculo $0,1 \%$, sino por enormes factores de 10 o más. Esto puede afectar considerablemente la química y la estructura térmica de la atmósfera superior.

Varios investigadores discutieron formas en las cuales los cambios en la atmósfera superior pueden influir sobre la superficie de la Tierra (Jackman et al., 2016). Isaac 
Held, de la Administración Nacional Oceánica y Atmosférica (National Oceanic and Atmospheric Administration o NOAA, por su acrónimo en idioma inglés), exploró esta observación con más detalle (Held, 2012). Él describió cómo es que la pérdida de ozono en la estratósfera podría alterar la dinámica de la atmósfera en las capas inferiores, ya que el enfriamiento de la estratósfera polar asociado con la pérdida de ozono incrementa el gradiente horizontal de temperatura cerca de la tropopausa, alterando el flujo de momento angular en los vórtices de latitudes intermedias. El momento angular es importante ya que el equilibrio del momento angular en la troposfera controla los vientos superficiales que se mueven hacia el Oeste.

Partiendo del hecho de que las variaciones de radiación ultravioleta asociadas al ciclo solar genera cambios allí donde se forma el ozono estratosférico, en la estratosfera tropical. Cuando hay poca actividad solar y se forma poco ozono allí se produce un enfriamiento que empuja la tropopausa hacia arriba acelerando la convección y activando la célula de Hadley. El resultado es un anticiclón subtropical mas potente que asciende en latitud, y que a su vez acelera los alisios. Dado que se ha de conservar el momento angular esta aceleración de los alisios introduce a su vez nuevos cambios en la circulación acelerando el chorro subtropical y la circulación polar. El resultado de ese cambio de patrón de la circulación, y debido básicamente a forzamientos dinámicos, consiste en un aumento muy significativo de la temperatura de verano cuando hay forzamiento solar y lo contrario. La descripción de los cambios de circulación de los últimos 50 años que se ha estudiado previamente puede estar relacionada con cambios en la actividad solar.

Basándose en el conocimiento de los cambios de actividad solar, se han calculado predicciones para posibles futuras emisiones solares. Se ha sugerido una cierta posibilidad de retorno a condiciones de mínimo de Maunder en los próximos 40 años (Ineson et al., 2015) por lo que puede convertirse en un experimento en tiempo real.

\section{REFERENCIAS}

CLIVAR-ESPAÑA Red temática. Clima en España: pasado, presente y futuro. Informe de evaluación del Cambio Climático Regional. p. 28 y 40. Enero de 2010.

De Castro, M., Martín-Vide, J., Alonso, S. (2015). El Clima de España: pasado, presente y escenarios del clima para el siglo XXI. En: J.M. Moreno (Ed.), Evaluación Preliminar de los impactos en España por efecto del cambio climático. Ministerio de Medio Ambiente. pp. 1-64. 2005. pp. 18 y 23.

Held, I. M. (2012). Issues in Climate Science Underlying Sun/Climate Research. National Research Council. Committee on the Effects of Solar Variability on Earth's Climate. Space Studies Board. Division on Engineering and Physical Sciences. The effects of solar variability on earth's climate A workshop report. THE NATIONAL ACADEMIES PRESS. Washington, D.C., p. 21.

Ineson, S., Maycock, A., Gray, L., Scaife1, A., Dunstone, N., Harder, J., Knight, J., Lockwood, M., Manners, J., Wood, R. Regional climate impacts of a possible future grand solar minimum. Nature communications, 6, Art. Number 7535, p. 1. Junio 2015. Disponible en: http://www.nature.com/naturecommunications 
Ineson, S., Scaife, A.,. Knight, J. Manners, J., Dunstone, N., Gray, L., and Haigh, J. Solar forcing of winter climate variability in the northern hemisphere, Nature Geoscience. Octubre 2011.

IPCC (2013). Climate Change 2013. The Physical Science Bases. TS.2, p. 37.

Jackman ,C.H. (2016). Atmospheric changes caused by galactic cosmic rays over the period 1960-2010, Atmos. Chem. Phys., 16, 5853-5866.

Kalnay et al (1996). The NCEP/NCAR 40-year reanalysis project, Bull. Amer. Meteor. Soc., 77, 437-470.

Lean, J. (2000). Evolution of the Sun's Spectral Irradiance since the Maunder Minimum. Geophys. Res. Lett. 27, 2425-2428.

Lomas González, A., Torrijo Murciano, R., “Análisis de la precipitación de otoño en varios observatorios durante las últimas décadas y su relación con cambios en la circulación atmosférica". XXXIV Jornadas del AME, Teruel. Marzo de 2016.

Lomas González, A., Torrijo Murciano, R., "Estudio sobre la evolución de la temperatura en verano en varios observatorios españoles y su relación con cambios en la circulación general atmosférica". XXXIII. Jornadas del AME, Oviedo, p. 3. Abril de 2014.

Sutton, R. and Dong, B. (2012). Atlantic Ocean influence on a shift in European climate in the 1990s. Nature Geoscience, 5, pp. 788-792. ISSN 1752-0908. p. 788. Disponible en: http://centaur.reading.ac.uk/30109/.

Woollings, T., M. Lockwood, G. Masato, C. Bell, and L. Gray (2010). Enhanced signature of solar variability in Eurasian winter climate. Geophys. Res. Lett., 37, L20805. Disponible en:

http://onlinelibrary.wiley.com/doi/10.1029/2010GL044601/full 